\title{
EVALUACIÓN DE HÍBRIDOS DE PAPAYA (Carica papaya L.) EN POCOCÍ, LIMÓN, COSTA RICA ${ }^{1}$
}

\author{
Eric Mora ${ }^{2}$, Antonio Bogantes ${ }^{3}$
}

\begin{abstract}
RESUMEN
Evaluación de híbridos de papaya (Carica papaya $\mathrm{L}$.) en Pococí, Limón, Costa Rica. Se estableció un experimento con nueve híbridos de papaya con el objetivo de determinar sus potenciales agronómicos y comerciales en una región de alta pluviosidad (cantón de Pococí, provincia de Limón). Las características evaluadas fueron tamaño de fruta, productividad total, brix de la pulpa y susceptibilidad a la antracnosis de la fruta causado por Colletotrichum gloesporioides. Se determinó que solo uno de los materiales mostró características similares al testigo comercial como fruta para consumo en fresco. Otro de los híbridos tuvo potencial para uso con fines agroindustriales.
\end{abstract}

\begin{abstract}
Evaluation of papaya (Carica papaya L.) hybrids in Pococí, Limón, Costa Rica. An experiment was established to determine the agronomic and commercial potential of nine papaya hybrids in a region of high rainfall (Pococí region, Limón province). The characteristics determined where fruit size, total productivity, pulp brix and susceptibility to fruit rot caused by Colletotrichum gloesporioides. It was determined that only one of these hybrids had commercial potential as a dessert fruit comparable to the control. One other hybrid had potential to be used for industrial purposes.
\end{abstract}

\section{INTRODUCCION}

En Costa Rica, el consumo per cápita de papaya es de tan solo $15,08 \mathrm{~kg}$, por debajo de otras frutas como banano, piña, sandía, naranja y manzana (PIMA 2003). Ese bajo nivel de consumo se debe básicamente a la pobre calidad organoléptica de las variedades cultivadas y trae como consecuencia que el mercado fácilmente se sature, lo que a su vez origina bajos precios para productores y una crisis casi permanente en este sector. Situaciones similares se dan con casi todas las frutas en todo el mundo. Como ejemplo, se puede citar el caso de la manzana, afectada también por una constante sobreproducción. A pesar de esto, el mercado de manzanas de calidad no está saturado (O Rourcke 2000, citado por Barrit 2000). Según Barrit (2000), el problema no es la sobreproducción de manzana, "sino la sobreproducción de tipos de manzana que el consumidor no quiere".

En otros países como Brasil, la introducción de nuevas variedades de papaya de buena calidad gustativa ha expandido fuertemente el desarrollo de este frutal durante los últimos 30 años (David y Gomes 2000).

Con el fin de estimular el consumo de esta fruta, así como de proveer a los agricultores de nuevas variedades para diversificar, el Instituto Nacional de Innovación y Transferencia en Tecnología Agropecuaria (INTA) y la Universidad de Costa Rica (UCR) han desarrollado en los último años algunos materiales con potencial comercial y de adaptación a las condiciones edafoclimáticas de la región Atlántica de Costa Rica.

\footnotetext{
1 Recibido para publicación el 9 de febrero del 2004.

2 Estación Experimental Fabio Baudrit, Universidad de Costa Rica. Alajuela, Costa Rica.

3 Estación Experimental Los Diamantes, INTA-MAG. Guápiles, Costa Rica.
} 
El objetivo de este trabajo fue el de comparar el potencial agronómico y comercial de nueve híbridos de papaya bajo las condiciones típicas de manejo de un productor de papaya en la región Atlántica de Costa Rica.

\section{MATERIALES Y MÉTODOS}

\section{Ubicación del experimento}

El experimento se realizó entre diciembre del 2001 y octubre del 2002 en la finca de un productor particular de papaya localizado en el distrito de La Rita, cantón de Pococí, provincia de Limón. La altura aproximada de dicha región es de $225 \mathrm{msnm}$. Los datos de precipitación mensual y temperatura diaria promedio en la zona durante el período evaluado se observan en la Figura 1.

\section{Tratamientos y diseño experimental}

Se evaluaron nueve híbridos distribuidos en un arreglo de bloques al azar con 3 repeticiones y 15 plantas por parcela.

Las variables evaluadas fueron: total de flores en tres axilas, fertilidad de las flores hermafroditas (\%), altura $(\mathrm{cm})$ a primera fruta, número y peso $(\mathrm{g})$ promedio de frutas, así como el brix de la pulpa y la severidad de antracnosis (Colletotrichum gloesporioides) al inicio y final de la primera cosecha. Esta severidad se calculó haciendo una observación visual de la fruta una vez que esta alcanzara un $100 \%$ de color amarillo externamente.

Las variables "altura de inserción de primera fruta" y "número de frutas" se midieron nueve meses después

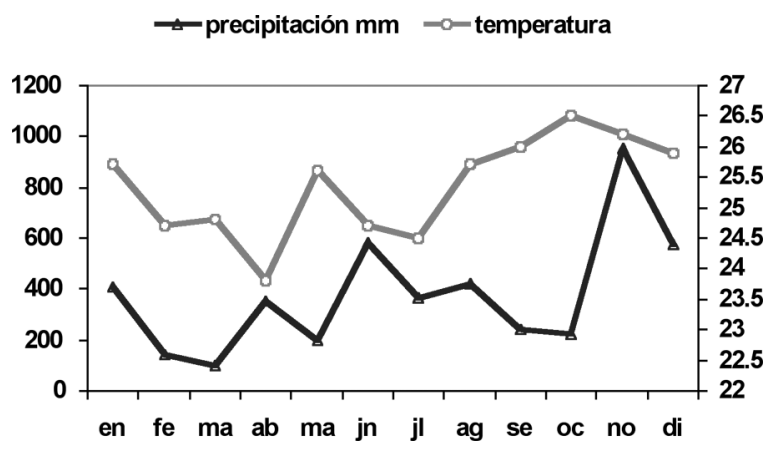

Figura 1. Datos de precipitación y temperatura diaria promedio, durante los meses del estudio. La Rita, Pococí, 2002. del transplante al campo, aproximadamente en la misma fecha de inicio de cosecha. La variable "número de flores por axila" y "porcentaje de flores fértiles" se midió tres meses después de iniciada la floración.

También se realizaron observaciones sobre la incidencia de carpelodia de las flores hermafroditas, así como la forma de la fruta y de su cavidad plancentaria.

\section{Origen de los híbridos}

Las líneas utilizadas para la producción de los híbridos tienen diferentes orígenes, tal y como se detalla a continuación:

a. Líneas T1 y LM: materiales seleccionados y estabilizados por los autores durante varios años a partir de genotipos cultivados en Costa Rica.

b. Línea ZM: material estable introducido de Cuba.

c. Líneas HC95-3, HC95-6, HC95-7, HC95-31 y MG: líneas generadas por hibridación y tres generaciones de autofecundación a partir de materiales autóctonos con introducciones de Hawaii (Mora y Bogantes 1999-2002).

Se utilizó como testigo el híbrido "Pococí" desarrollado durante el período de 1995 al 2001 y recientemente validado a través del convenio UCR-INTA.

\section{Manejo agronómico}

Se sembró a 2,5 m entre hileras y 2,5 m entre planta para una densidad de 1.600 plantas por ha.

Se aplicó el manejo del productor cuyo paquete principal consistió en:

Fertilización: $246 \mathrm{~kg}$ de nitrógeno, $142 \mathrm{~kg}$ de fósforo y $280 \mathrm{~kg}$ de potasio por hectárea durante los primeros doce meses de cultivo. Las fuentes utilizadas fueron las fórmulas comerciales 10-30-10, 18-5-15-61.2 y 15-3-31 fraccionadas mensualmente. Lo anterior se complementó con la aplicación de fertilizantes foliares a base de calcio y boro cada dos meses.

Protección: Se realizaron aplicaciones preventivas y de control de antracnosis (Colletotrichum gloesporioides) en fruta a base de benomil y mancozeb. El combate del díptero Toxotrypana curvicauda se hizo a base de los insecticidas dimetoato y cipermetrina. El control de malezas se hizo con glifosato. 


\section{RESULTADOS Y DISCUSIÓN}

\section{Características fisiológicas florales y número de frutas:}

El número de frutas por híbrido osciló entre 22.44 y 41.78 (Cuadro 2), es decir, una diferencia casi del $50 \%$. Los dos materiales con el menor número de frutas (T1XHC95-7 y T1XZM) presentaron también los mayores pesos promedios por fruta, evidenciando un efecto de compensación.

Como se puede observar en el Cuadro 1, la incidencia de esterilidad femenina de las plantas hermafroditas sobre la productividad fue muy baja, pues variedades con bajos porcentajes de flores fértiles presentaron mayor número de frutas que otras con mayor fertilidad. Este problema tradicionalmente se ha asociado con bajas productividades (Nakasone 1967). Sin embargo, los datos de este trabajo demuestran que esto no es siempre cierto. Debe recordarse que casi todas las variedades de papaya producen varias flores por axila. Debido al raleo natural producto de la partición asimétrica de carbono por la competencia entre primordios florales, un alto número de flores fértiles no necesariamente se traduce en un gran número de frutas. Por este motivo, una variedad que tienda a producir una sola flor fértil por axila puede tener una productividad final igual que otra que produce varias flores fértiles. Sin embargo, debido a que la esterilidad femenina en papaya se debe a una interacción genotipo-ambiente (Storey 1953), una baja propensión genética a este fenómeno podría garantizar una mayor estabilidad en la producción bajo diversos regímenes climáticos. De todas formas, ninguno de los híbridos mostró niveles de esterilidad que comprometieran sus potenciales de producción bajo las condiciones del estudio.

Cuadro 1. Flores totales ( 3 axilas por planta) y porcentaje de flores fértiles de plantas hermafroditas en los híbridos evaluados. Pococí, 2002.

\begin{tabular}{lcc}
\hline \multicolumn{1}{c}{ Híbridos } & Fertilidad/axila (\%) & \# Flores/3 axilas \\
\hline T1 X ZM & $75,55 \mathrm{a}^{1}$ & $11,00 \mathrm{ab}$ \\
LM X HC95-6 & $75,22 \mathrm{a}$ & $9,55 \mathrm{~b}$ \\
T1 X HC95-7 & $67,33 \mathrm{ab}$ & $11,33 \mathrm{ab}$ \\
MG X HC95-7 & $53,44 \mathrm{bc}$ & $11,44 \mathrm{ab}$ \\
HC95-3 X HC95-7 & $49,88 \mathrm{c}$ & $10,88 \mathrm{ab}$ \\
MG X HC95-6 & $43,55 \mathrm{c}$ & $11,11 \mathrm{ab}$ \\
HC95-3 X HC95-6 & $43,44 \mathrm{c}$ & $12,22 \mathrm{a}$ \\
Testigo & $41,11 \mathrm{c}$ & $12,11 \mathrm{a}$ \\
MG X HC95-31 & $39,22 \mathrm{c}$ & $11,22 \mathrm{ab}$ \\
\hline
\end{tabular}

1 Tratamiento con igual letra en una misma columna presentan diferencias no significativas según Prueba de Tukey $5 \%$.

\section{Productividad total}

Las productividades de los primeros 7 híbridos fueron muy satisfactorias si se comparan con las referencias para otras variedades híbridas que se consiguen en el mercado internacional con tamaños de fruto similares, tales como lo híbridos taiwaneses Tainung \#1 y Tainung \#2, cuyas productividades rondan los 50 a 60 toneladas/ha/año (David y Gomez, 2000).

El híbrido MG X HC95-6 tuvo la mayor productividad total con un estimado por hectárea de $108.836 \mathrm{~kg}$ (Cuadro 2). Esta alta productividad se debió básicamente a la combinación de un tamaño de fruta relativamente grande $(1.756 \mathrm{~kg})$, y a un aceptable número total de frutas.

Cuadro 2. Altura del suelo a primera fruta, número y peso promedio de frutas evaluadas para los primeros 5 meses de cosecha. Pococí, 2002.

\begin{tabular}{llllll}
\hline Híbridos & $\begin{array}{c}\text { Altura a } \\
\text { primera } \\
\text { fruta (cm) }\end{array}$ & $\begin{array}{c}\text { Número } \\
\text { frutos }\end{array}$ & $\begin{array}{c}\text { Peso/ } \\
\text { fruto (g) }\end{array}$ & $\begin{array}{c}\text { Peso/ } \\
\text { árbol (kg) }\end{array}$ & $\begin{array}{c}\text { Peso/ha } \\
(\mathbf{k g})\end{array}$ \\
& & & & & \\
\hline & & & & & \\
T1 X HC95-7 & $60 \mathrm{~cd}^{1}$ & $22,44 \mathrm{c}$ & $2.089 \mathrm{ab}$ & $46,90 \mathrm{ab}$ & $75.040 \mathrm{ab}$ \\
T1 X ZM & $60,78 \mathrm{bcd}$ & $23,22 \mathrm{c}$ & $2.533 \mathrm{a}$ & $58,67 \mathrm{ab}$ & $93.867 \mathrm{ab}$ \\
Testigo & $72,56 \mathrm{ab}$ & $41,78 \mathrm{a}$ & $1.294 \mathrm{~cd}$ & $54,19 \mathrm{ab}$ & $86.711 \mathrm{ab}$ \\
LM X HC95-6 & $67,44 \mathrm{bc}$ & $34,33 \mathrm{ab}$ & $1.211 \mathrm{~cd}$ & $40,71 \mathrm{~b}$ & $65.138 \mathrm{~b}$ \\
MG X HC95-6 & $54,67 \mathrm{~d}$ & $37,78 \mathrm{ab}$ & $1.756 \mathrm{bc}$ & $68,02 \mathrm{a}$ & $108.836 \mathrm{a}$ \\
MG X HC95-31 & $62,89 \mathrm{bcd}$ & $30 \mathrm{bc}$ & $1.428 \mathrm{~cd}$ & $42,88 \mathrm{ab}$ & $68.613 \mathrm{ab}$ \\
MG X HC95-7 & $53,33 \mathrm{~d}$ & $34,89 \mathrm{ab}$ & $1.450 \mathrm{bcd}$ & $50,5 \mathrm{ab}$ & $80.800 \mathrm{ab}$ \\
HC95-3 X & & & & & \\
HC95-6 & $82,00 \mathrm{a}$ & $34,78 \mathrm{ab}$ & $1.072 \mathrm{~d}$ & $36,79 \mathrm{~b}$ & $58.862 \mathrm{~b}$ \\
HC95-3 X & & & & & \\
HC95-7 & $67,11 \mathrm{bc}$ & $33,67 \mathrm{ab}$ & $1.067 \mathrm{~d}$ & $36,11 \mathrm{~b}$ & $57.778 \mathrm{~b}$
\end{tabular}

1 Tratamiento con igual letra en una misma columna presentan diferencias no significativas según Prueba de Tukey $5 \%$.

El híbrido T1 X ZM mostró también un alto potencial de producción por hectárea $(93867 \mathrm{~kg})$, lo cual, sumado al gran tamaño de su fruta, le confieren potencial para ser utilizado como variedad con fines agroindustriales.

A pesar de poseer una fruta mediana $(1,3 \mathrm{~kg})$, el testigo "Pococî", se ubicó entre los tres materiales más productivos debido a su alto número de frutas por planta (43), lo cual lo confirma como una variedad atractiva para el productor desde el punto de vista económico.

En el otro extremo se ubican los híbridos HC95-3 X HC95-6 y HC95-3 X HC95-7, con una producción por hectárea menor a las 60 toneladas, producto de la 
combinación de pocas frutas con poco peso. El pobre comportamiento de estos dos híbridos se puede explicar en parte debido a su cercanía filogenética, lo cual incide en una baja manifestación de heterosis. Ambos materiales son producto de la segregación de un mismo híbrido.

\section{Altura de producción}

No se presentaron diferencias significativas en cuanto a altura de producción, ya que la mayor diferencia entre los materiales de los extremos del rango total fue de apenas 29 centímetros. Aún cuando una baja altura de producción puede ser positivo por estar asociado a precocidad (Storey 1953) y mayor facilidad inicial de cosecha, también tiene sus desventajas. En condiciones de alta pluviosidad como las prevalecientes en la zona donde se realizó este trabajo, el salpique de la lluvia sobre el suelo favorece la diseminación del inóculo de Phytophtora sp. sobre la fruta, dando origen a severas epidemias de este patógeno comunes en la región. Por este motivo, las alturas de inicio de fructificación deberían ser mayores a los $80 \mathrm{~cm}$ preferiblemente. Por otra parte, una excesiva precocidad también puede estar asociado a una vida útil menor de la plantación (Mora y Bogantes 1999-2002).

\section{Tamaño de fruta:}

Como se puede observar en el Cuadro 1, las frutas de la mayoría de los híbridos se encuentran dentro del rango de 1 a 1,5 kg. Según Giacometti (1987) el tamaño preferido por los consumidores para mercados asiáticos y latinoamericanos es de $1,5 \mathrm{~kg}$. mientras que para los mercados de exportación es de alrededor de 0,5 $\mathrm{kg}$. Aún cuando casi todas las variedades en nuestro país producen frutas con pesos cercanos de los dos kilos, el mercado nacional también acepta frutas entre 1 y
$1,5 \mathrm{~kg}$ (Mora y Bogantes 1999-2002). Este tamaño es bastante adecuado para las necesidades de los consumidores, pues facilita su transporte y almacenamiento, así como su consumo en un corto período de tiempo.

Contrario a lo anterior, entre los productores e intermediarios nacionales, existe una tendencia a preferir las variedades de fruta cercanas a los dos kilos, pues implica menos trabajo durante las labores de cosecha y embalaje para una cantidad determinada de peso. Sin embargo, para el consumidor, esto implica un alto costo por fruta, y un posible desperdicio si ese tamaño excede sus posibilidades de consumo. En los mercados actuales tan competitivos, el éxito comercial de un producto depende cada vez más en ajustarse y satisfacer a las demandas de los consumidores (Martinez y Davis 2002).

Por su parte, el híbrido T1XZM con un peso promedio cercano a los $2,5 \mathrm{~kg}$, es más apto para la actividad industrial.

\section{Cavidades y forma de los frutos}

En papaya, la cavidad central de su fruta puede variar desde profundamente estrellada hasta completamente redonda. Para consumo fresco este es un factor importante, pues la cavidad redonda facilita la extracción de la semilla haciendo más fácil su consumo (Giacometti 1987). De igual forma, esta característica es también importante en fruta para industria, donde la eficiencia del procesado disminuye los costos operativos. Prácticamente todos los híbridos presentaron una cavidad menos estrellada que la variedad "Sunrise" o "hawaiana" (Figura 2).

Tradicionalmente, la papaya "Sunrise" ha impuesto la noción en el mercado internacional que su forma

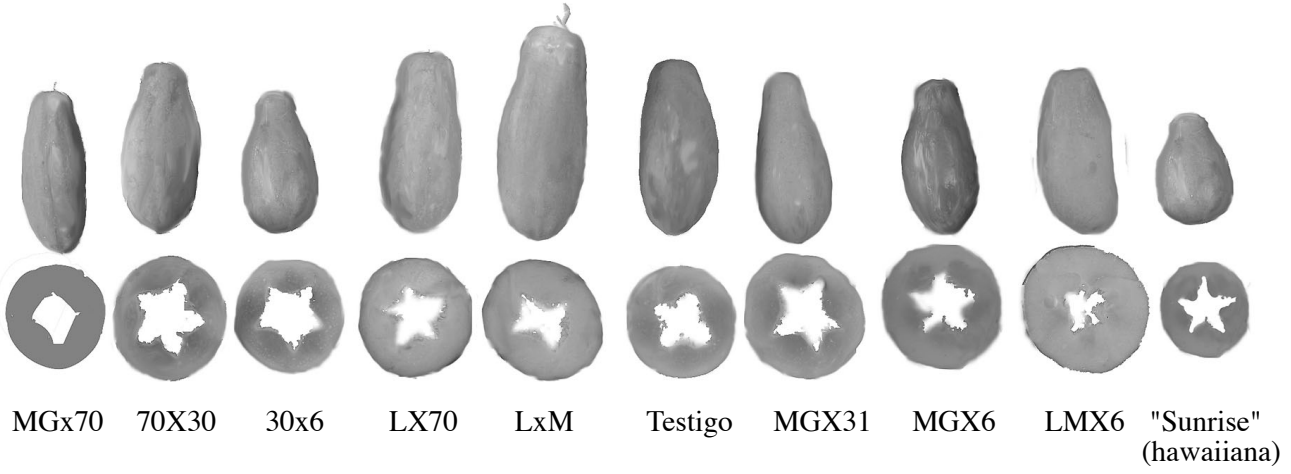

Figura 2. Formas típicas de las frutas evaluadas y sus respectivas cavidades placentarias (corte transversal). Orden de aparición de izquierda a derecha: MGXHC95-7, HC95-7XHC95-3, HC95-30X HC956, T1XHC95-7, T1XZM, Testigo, MGXHC95-31, MGXHC95-6, LM X HC95-6 y "Sunrise" (como comparador). 
piriforme es la más adecuada. Sin embargo, el mercado actual parece preferir una fruta redonda pues facilita su consumo con cuchara (Nakasone, citado por Giacometti 1987). La mayor cantidad de los híbridos evaluados presentó una forma intermedia, con un "cuello" menos acentuada que las variedades hawaiianas (Figura 2).

\section{Brix:}

En Costa Rica, los materiales tradicionales generalmente se sitúan entre los 8,5 y 10 grados brix. Los brix obtenidos con estos híbridos son relativamente más altos a los de estas variedades. Sin embargo, no llegaron a niveles más adecuados por encima de los 11,5, el cual ha sido establecido como el mínimo aceptable para las variedades tipo hawaiano (Paull, citado por Zhou et al. 2000). Debe recordarse que la alta pluviosidad por encima de los $4000 \mathrm{~mm}$ anuales prevaleciente en la zona donde se realizó este trabajo (Figura 1) probablemente afectó esta característica. No obstante, esta región se seleccionó por ser la más representativa de este frutal en nuestro país en términos de área sembrada.

Con excepción de los materiales HC95-3 X HC957 y LM X HC95-6, los demás materiales mantuvieron

Cuadro 3. Brix de la pulpa y porcentaje de área afectada de fruta por antracnosis de los híbridos evaluados al inicio y al final de la primera cosecha. Pococí 2002.

\begin{tabular}{|c|c|c|c|c|}
\hline $\begin{array}{r}\text { Híbridos } \\
\text { E }\end{array}$ & \multicolumn{2}{|c|}{${ }^{\mathbf{o}}$ Brix } & \multicolumn{2}{|c|}{ Antracnosis (\%) } \\
\hline $\begin{array}{l}\text { Testigo } \\
\text { MG X }\end{array}$ & $10,64 \mathrm{ab}^{1}$ & $11,37 \mathrm{a}$ & $4,55 \mathrm{a}$ & $0,63 \mathrm{~b}$ \\
\hline $\begin{array}{l}\text { HC95-6 } \\
\text { MG X }\end{array}$ & $11,07 \mathrm{a}$ & $11,19 \mathrm{ab}$ & $9,33 \mathrm{a}$ & $3,67 \mathrm{~b}$ \\
\hline $\begin{array}{l}\text { HC95-7 } \\
\text { MG X }\end{array}$ & $10,07 \mathrm{abc}$ & $10,60 \mathrm{ab}$ & $7,40 \mathrm{a}$ & $8,67 \mathrm{ab}$ \\
\hline $\begin{array}{l}\text { HC95-31 } \\
\text { T1 X }\end{array}$ & $10,66 \mathrm{ab}$ & $10,50 \mathrm{ab}$ & $1,44 \mathrm{a}$ & $5,08 \mathrm{~b}$ \\
\hline $\begin{array}{l}\text { ZM } \\
\text { LM X }\end{array}$ & $10,76 \mathrm{ab}$ & $10,47 \mathrm{ab}$ & $11,2 \mathrm{a}$ & $3,67 \mathrm{~b}$ \\
\hline $\begin{array}{l}\text { HC95-6 } \\
\text { HC95-3 X }\end{array}$ & $\mathrm{X}^{8,84 \mathrm{c}}$ & $10,34 \mathrm{ab}$ & 14,11 a & $5,82 \mathrm{~b}$ \\
\hline $\begin{array}{l}\text { HC95-6 } \\
\text { T1 X }\end{array}$ & $10,49 \mathrm{ab}$ & $10,14 \mathrm{ab}$ & $8,80 \mathrm{a}$ & $5,82 \mathrm{~b}$ \\
\hline $\begin{array}{l}\text { HC95-7 } \\
\text { HC95-3 X }\end{array}$ & $\mathrm{X}^{9,44 \mathrm{bc}}$ & $9,87 \mathrm{bc}$ & 14,89 a & $19,5 \mathrm{a}$ \\
\hline HC95-7 & $10,07 \mathrm{abc}$ & $8,53 \mathrm{c}$ & $10,07 \mathrm{a}$ & $12,2 \mathrm{ab}$ \\
\hline
\end{tabular}

1 Tratamiento con igual letra en una misma columna presentan diferencias no significativas según Prueba de Tukey 5\%. un comportamiento estable entre el primero y segundo muestreo, con variaciones menores a un grado brix. En las variedades tradicionales cultivadas en nuestro país, es común encontrar variaciones importantes entre las frutas cosechadas al inicio del período productivo con aquellas cosechadas unos meses después, independientemente de las condiciones climáticas prevalecientes. Estas diferencias posiblemente están relacionadas con las fluctuaciones de la relación fuente-sumidero que se da a través del ciclo, la cual se sabe puede afectar los niveles de azúcares en papaya (Zhou et al. 2000).

\section{Antracnosis}

Los porcentajes de área de infección para los híbridos "Pococí" (testigo), MG X HC95-6, MG X HC9531, MG X HC95-7 y HC95-3 X HC95-6 son comparables a los obtenidos por Solano y Arauz (1995) para materiales criollos en la misma zona de evaluación (cantón de Pococí). Dichos autores evaluaron diferentes productos químicos para el control de antracnosis y obtuvieron porcentajes de área afectada cercanas al 5\% con los mejores tratamientos evaluados. Por su parte, los híbridos T1 X HC95-7, MG X HC95-7 y HC95-3 X HC95-7 presentaron porcentajes relativamente más altas (entre el $10 \%$ y el $20 \%$ ) pero no significativamente diferentes.

Estos datos evidencian que posiblemente ninguno de los híbridos sea más resistente a la antracnosis que los materiales criollos. Sin embargo, el hecho de que algunos de ellos tampoco evidencien mayor susceptibilidad, sumado a sus bondades organolépticas, les confiere mayor potencial comercial que las variedades tradicionales.

\section{CONCLUSIONES}

De los nueve materiales evaluados destacan el testigo "Pococî" y el híbrido MG X HC95-6. El segundo material presenta un tamaño de frutas relativamente más grande $(1.75 \mathrm{~kg}$ vs $1.29 \mathrm{~kg})$ pero los brix de ambos son muy similares, cercanos a los 11. El rendimiento total por hectárea fue alto en ambos híbridos, pero el MG X HC95-6 supera en cerca de un $20 \%$ al testigo. Lo anterior se debe básicamente al mayor tamaño promedio de sus frutas en vista de que el número de éstos es prácticamente igual en ambos casos. La susceptibilidad a la antracnosis es similar entre los dos materiales y comparable a la susceptibilidad de las variedades tradicionales de la zona. Por los motivos anteriores, debe continuarse la evaluación del potencial 
comercial y agronómico del híbrido MG X HC95-6 a través de siembras en escalas mayores.

El híbrido T1 X ZM tiene potencial comercial para industria debido a su gran tamaño, alta productividad, brix aceptable, forma homogénea y cavidad placentaria relativamente pequeña.

Los demás híbridos pueden descartarse de futuras evaluaciones debido a que no reúnen una combinación de caracteres favorables como para justificar un interés comercial potencial.

\section{LITERATURA CITADA}

BARRIT, B.H. 2001. Apple quality for consumers. The Compact Fruit Tree. 34(2): 54-56.

DAVID S.L.; GOMES, J.A. 2000. Cultura do mamao. Ed. por Ronaldo de Oliveira Sales. Fortaleza, Brasil. 57p. Disponible en: http://www.ediho.es/horticom/tem aut/cd/brasil/mamao.pdf

GIACOMETTI, D.C. 1987. Papaya breeding. Acta Horticulturae 196:53-60.
MARTINEZ, S.; DAVIS, D. 2002. Farm business practices coordinate production with consumer preferences. Food Review 25(1):33-38.

MORA, E.; BOGANTES, A. 1999-2002. Evaluación del potencial comercial de líneas S2 de papaya (Carica papaya L.) en la Región Atlántica de Costa Rica. Revista de Agricultura Tropical 32: 73-80.

NAKASONE, H.Y. 1967. Papaya breeding in Hawaii. Agronomía Tropical 17:391-397.

PIMA. 2003.Tendencias del consumo de frutas, hortalizas y pescado en las familias de Costa Rica. CENADA, Costa Rica. 48 p.

SOLANO, V.; ARAUZ, L.F. 1995. Combate de antracnosis en frutos de papaya mediante aplicaciones de fungicidas en el campo en la zona Atlántica de Costa Rica. Agronomía Costarricense 19(2):25-30.

STOREY, W.B. 1953. Genetics of papaya. Journal of Heredity (44):70-78

ZHOU, L.; CHISTOPHER, D.; PAULL, R. 2000. Defoliation and fruit removal effects on papaya fruit production, sugar metabolism, and sucrose metabolism. J. Amer. Soc. Hort. Sci. 125(5):644-652 